\title{
A autonomia na adolescência: Revisando conceitos, modelos e variáveis.
}

\author{
Paola Vargas Barbosa \\ Adriana Wagner \\ Universidade Federal do Rio Grande do Sul
}

\begin{abstract}
Resumo
A conquista da autonomia está descrita como uma importante tarefa desenvolvimental da adolescência. Todavia, a complexidade da temática dificulta a compreensão do que seja a autonomia, assim como a comparação dos resultados encontrados na literatura científica. Com o objetivo de descrever os principais conceitos, modelos teóricos e variáveis associadas à autonomia adolescente, realizou-se uma revisão sistemática das publicações brasileiras e internacionais. Selecionaram-se 46 artigos que tratassem da autonomia como capacidade pessoal. Foram classificados de acordo com: conceitos utilizados, amostra, instrumentos e variáveis associadas à autonomia. A análise revelou uma diversidade de conceitos e instrumentos para compreensão do fenômeno; a existência de um perfil de adolescente no qual se baseia grande parte das publicações; a associação desta habilidade a variáveis contextuais, de ajustamento e clima familiar. Percebeu-se a descrição da autonomia como complexa e marcada por variáveis contextuais. Uma discussão critica sobre a produção encontrada na área foi feita.
\end{abstract}

Palavras-chave: autonomia; adolescente; adolescência.

\begin{abstract}
Autonomy in adolescence: Reviewing concepts, models and variables. The development of autonomy is described as an important developmental task during adolescence. However, the complexity of the theme hinders the comprehension of what is considered autonomy, as the relation of the results that are found in the scientific literature. With the objective of describing the main concepts, theoretical models and variables associated to adolescent's autonomy, was done a systematic review of Brazilian and international publications. 46 papers that discussed the autonomy as a personal ability were selected. They were classified according to: concepts used, sample, instruments and variables associated to autonomy. The analysis revealed a multiplicity of concepts and instruments in the comprehension of this ability; the existence of a main profile of adolescent in which are based most of the publication; the association of this capacity to contextual, adjustment and family environment variables. The autonomy was described as a complex and influenced by contextual variables. A critical discussion about what has been produced in the field was made.
\end{abstract}

Keywords: autonomy; adolescent; adolescence.

\section{Resumen}

La autonomía en la adolescencia: Revisión de conceptos, modelos y variables. La conquista de la autonomía se describe como una tarea importante en el desarrollo de la adolescencia. Sin embargo, la complejidad de la materia hace que sea difícil de comprender lo que es la autonomía, así como la comparación de los resultados en la literatura científica. Con el fin de describir los principales conceptos, modelos teóricos y las variables asociadas a la autonomía de los adolescentes, se realizó una revisión sistemática de publicaciones nacionales e internacionales. Fueron seleccionados 46 artículos que abordan la autonomía como capacidad personal. Fueron clasificados de acuerdo a: los conceptos utilizados, la muestra, los instrumentos y las variables asociadas con la autonomía. El análisis reveló una diversidad de conceptos y herramientas para comprender el fenómeno; la existencia de un perfil de los jóvenes en el que gran parte de las publicaciones se basa; la combinación de esta habilidad a las variables contextuales, de ajuste y de ambiente familiar. La autonomía se describe como compleja, y marcada por variables contextuales. Se realizó una discusión crítica sobre la producción científica revisada.

Palabras clave: autonomía; adolescente; adolescencia. 
$\mathrm{O}$ processo de desenvolvimento adolescente é marcado por importantes transições. A conquista paulatina da autonomia tem sido descrita como uma das mais importantes tarefas desenvolvimentais dessa fase (Celen Cok, Bosma, \& Djurre, 2006). Porém, o que significa tornar-se autônomo?

Na psicologia, o conceito da autonomia tem sido estudado e discutido a partir de diferentes referenciais teóricos. De forma geral, este construto é compreendido como complexo. Entendese que seu desenvolvimento é contínuo (Anderson, Worthington, Anderson, \& Jennings, 1994) e que tal capacidade é desenvolvida de forma diferente em dimensões distintas, como por exemplo, emocional, cognitiva ou funcional (Yeh \& Yang, 2006). Estudos dos anos 80 demonstraram que o seu desenvolvimento está positivamente correlacionado à idade (Noom, Dekovic, \& Meeus, 2001), que acontece de forma diferente para os meninos e meninas (Steinberg \& Silverberg, 1986) e que está associada ao bem estar psicológico (Lekes, Gingras, Philippe, Koestner, \& Fang, 2010). Além disso, aparece na literatura como sendo influenciada pelos estilos parentais (Baumrind, 1966), pelo nível educacional dos pais (Romich, Lundberg, \& Tsang, 2009) pelo contexto sociocultural do sujeito (Yeh \& Yang, 2006), pelo estilo de apego (Jamabo \& Jamabo, 2010), dentre inúmeras outras variáveis que se associam ao fenômeno.

Atualmente, as discussões registradas na literatura a respeito dessa temática circulam, principalmente, em torno de três aspectos. O primeiro, diz respeito ao conceito de autonomia. Essa capacidade tem sido concebida e descrita como formada por subconstrutos ou dimensões, numa tentativa de circunscrever a complexidade do tema. Alguns exemplos das dimensões relatadas para conceituar a autonomia foram: autonomia emocional (Steinberg \& Silverberg, 1986), cognitiva (Beckert, 2007), comportamental (Bakken \& Bronw, 2010) entre outras. A existência de diversos pontos de vista teóricos e ênfases em dimensões diferentes dessa habilidade tornam a pesquisa empírica nessa área muito heterogênea e de difícil análise.

Outra discussão recorrente nessa temática diz respeito ao desenvolvimento da autonomia. Pesquisadores têm discutido duas grandes visões sobre esse processo desenvolvimental. De um lado, numa perspectiva sociodinâmica, estudiosos prevêem que a estruturação da autonomia se dá num processo de gradual afastamento ou separação emocional das figuras de referência (como os pais ou responsáveis) (Bloss, 1994; Steinberg \& Silverberg, 1986). Dessa forma, a autonomia pode ser analisada como o extremo oposto da dependência emocional em relação aos progenitores. De outro lado, pesquisadores do desenvolvimento e psicólogos sociais definem a autonomia como uma habilidade que se desenvolve a partir de uma relação próxima e afetuosa com as figuras de referência (Deci \& Ryan, 2000; Yeh \& Yang, 2006). À luz dessa perspectiva, o contraponto da autonomia não é a dependência, mas sim a heteronomia, definida como a sujeição da pessoa à vontade de terceiros (Soenens et al., 2007).

Pesquisadores apontam que a conceituação da autonomia como a capacidade de independência em relação aos outros, acaba por reforçar a noção de que seu desenvolvimento se dá de forma individual, sendo assim concebida como uma capacidade contrária à relação ou ligação com outros (Kagitçibasi, 2005).
A partir desse ponto de vista, essa visão individualista do desenvolvimento da autonomia é consequência da construção de conhecimento enviesado pela cultura individualista ocidental, que julga, a partir de seus padrões e valores culturais, parâmetros universais. Pesquisadores dessa temática na perspectiva da psicologia cultural referem que a autonomia é valorizada também em culturas coletivistas (que não tem a independência como um valor proeminente). Entretanto nesses contextos, essa habilidade se desenvolve na relação de proximidade dos pais e familiares (Kagitçibasi, 2005).

Outro aspecto importante desse fenômeno diz respeito às inúmeras variáveis apontadas como associadas ou preditoras do desenvolvimento da autonomia. Pesquisas têm buscado demonstrar as diferenças no desenvolvimento da autonomia entre amostras com valores culturais diferentes, analisando culturas individualistas ou coletivistas (Ferguson, Kasser, \& Jahng, 2010); verificar as variações observadas por idade (Daddis, 2011), por gênero (Marsh, McFarland, Allen, McElhaney, \& Land, 2003) e por nível socioeconômico (Allen, Marsh, et al., 2002). Além disso, com o objetivo de mapear diferentes fatores associados a esse construto, a autonomia tem sido correlacionada com o clima familiar (Fuhrman \& Holmbeck, 1995), suporte parental (Lekes et al., 2010), legitimidade da autoridade parental (Cumsille, Darling, \& Martínez, 2010; Daddis, 2011) e outras variáveis que dizem respeito ao contexto. Algumas consequências do comportamento também aparecem na literatura como correlacionadas a altos ou baixos índices de autonomia. Entre elas: os problemas de comportamento, externalização (Eichelsheim et al. 2010), autoestima, bem estar (Yeh \& Yang, 2006), depressão e ideação suicida (Pace \& Zappulla, 2010), uso de substâncias (Scholte, Van Lieshout, \& Van Aken, 2001) e desempenho acadêmico (Smetana, Campione-Barr, \& Daddis, 2004).

Frente à complexidade da temática e sua multiplicidade de vertentes, nesse trabalho objetiva-se descrever os achados sobre autonomia a partir de uma revisão sistemática da literatura sobre o assunto.

\section{Método}

A presente revisão sistemática teve como questão norteadora a pergunta: "Quais são os conceitos utilizados em publicações científicas que descrevem a autonomia adolescente?". A partir desse questionamento pretendeu-se conhecer o conceito de autonomia, os processos de seu desenvolvimento e as principais variáveis envolvidas nos estudos realizados nos últimos 20 anos no exterior e dos últimos 15 anos no Brasil.

Fez-se um levantamento bibliográfico a partir de uma busca nas seguintes bases de dados: Academic search premier (EBSCO), Annual reviews, Cambridge Journals on line, Gale Academic onefile, General Science Fulltext (Wilson), Oxford Journals, Scielo.org, Science Direct (Elsevier), Web of Science, Wiley online Library e Springer link, disponibilizadas através do portal Periódicos da Capes. Além disso, para uma maior abrangência das publicações no Brasil, foi feita uma busca no site BVS Psicologia ULAPSI - Brasil, acessando as bases de dados Index Psi, LILACS e o Portal Nacional BVS Brasil em Saúde.

O levantamento teve como descritores as palavras 
"autonomia" e "adolescente (em português, inglês e espanhol), incluindo os artigos publicados em periódicos nacionais ou internacionais. A partir desses critérios foram encontrados 789 artigos. Através da leitura dos títulos e resumos foram excluídos artigos que não discutissem a autonomia como habilidade ou capacidade pessoal e que pudessem ser acessados na íntegra, totalizando uma seleção de 46 publicações. Todos os artigos selecionados foram lidos na íntegra e, a partir dessa leitura detalhada, foi construído um formulário para a organização das informações. Esse formulário continha dados de identificação do artigo (títulos, autores, ano de publicação e revista publicada), os participantes, objetivos, delineamento, principais resultados, conceito de autonomia adotado, instrumentos utilizados, e outras variáveis associadas à autonomia.

\section{Resultados}

Depois da leitura dos 46 artigos, 37 foram classificados como principais e nove como periféricos, nos quais a autonomia aparecia como um resultado observado e não como o objetivo do estudo. A maioria dos artigos selecionados estava na língua inglesa e apenas um artigo principal estava em português (Reichert \& Wagner, 2007). Apenas três artigos haviam sido publicados no Brasil.

\section{Participantes estudados}

Todas as 46 publicações selecionadas, com exceção de dois artigos, tinham os adolescentes como foco do estudo. Os principais critérios de inclusão dos participantes foram: série escolar, idade, raça, identidade cultural e situação de risco. Os participantes tinham idades entre a early adolescence (10-13 anos/16 artigos), middle adolescence (14-17 anos/ 20 publicações) e late adolescence (18- 21/4 artigos). Os seis artigos restantes (do total de 46 artigos) não especificaram a idade de seus participantes. A maioria dos adolescentes era Estadunidense (em 26 publicações), de um total de 12 países identificados. Não há menção na maioria dos trabalhos quanto ao nível socioeconômico e a identidade racial dos participantes. Dos trabalhos que o fazem, a maior parte dos sujeitos é branca e de classe média.

\section{Conceitos}

A autonomia tem sido um tema de interesse crescente na literatura, mas a multiplicidade e falta de clareza nos conceitos utilizados pulveriza os resultados encontrados, dificultando a construção de teorias consistentes sobre esse fenômeno. Apenas 20 dos 46 artigos analisados apresentam o conceito que utilizam sobre a autonomia. Nos demais, é possível apenas inferir, com base na discussão e literatura apresentada, o que entendem por autonomia.

Dos artigos analisados, 62,8\% deles utiliza a autonomia comportamental como conceito principal. A autonomia comportamental é apresentada como o processo de tomada de decisão, ou a capacidade de regular o próprio comportamento (Haase, Tomasik, \& Silbereisen, 2008; Peterson, Bush, \& Supple, 1999). Em outros trabalhos, a tomada de decisão é utilizada apenas como indicador da autonomia comportamental (Seiffge-
Krenke \& Pakalniskiene, 2011; Smetana \& Gettman, 2006; Wray-Lake, Crouter, \& McHale, 2010).

A autonomia comportamental ainda é descrita como uma capacidade consciente de tomar decisões em direção a um fim (Soenens et al., 2007). Tal conceito se baseia na Teoria da Autodeterminação (Deci \& Ryan, 2000), entendendo a autonomia não como independência, mas como função volitiva. Outras nomenclaturas utilizadas foram: autonomia de decisão (Scabini, 2000), independência (Blair \& Fletcher, 2011; Butner et al., 2009; Sellers, Black, Boris, Oberlander, \& Myers, 2011; Slater, Kelly, Lawrence, Stanley, \& Comello, 2011) e autonomia individual (Fuligni, 1998).

Em nove outros artigos, o conceito de Autonomia Emocional é utilizado. Nessas pesquisas a autonomia é descrita como a capacidade de perceber a si mesmo e aos pais como indivíduos separados, compreendendo-os como pessoas falíveis e normais (Blos, 1979). Tal descrição é também utilizada por Steinberg e Silverber (1986) na Escala de Autonomia Emocional (EAE), construída com o objetivo de incorporar o conceito de Blos à pesquisa empírica (Beyers et al., 2005).

Outro conceito que aparece relacionado com a autonomia emocional é o de separação (detachment). Tal discussão é feita a partir de críticas à EAE. Para os autores da escala, altos índices de afastamento dos pais (obtidos através de altos índices na escala) retratariam maior autonomia e se associariam com consequências positivas para o desenvolvimento adolescente, como melhor ajustamento (Steinberg \& Silverberg, 1986). Porém, algumas críticas foram apresentadas a essa perspectiva, quando altos índices na escala aparecem associados a sentimentos de insegurança em relação aos pais (Ryan \& Lynch, 1989) e sentimentos de depressão e ansiedade (Beyers \& Goossens, 2005). Para esses autores, a EAE seria uma medida de separação extrema (detachment) e não de autonomia emocional. Dessa forma, no contexto dos artigos aqui revisados, alguns utilizam o conceito de autonomia emocional (e a EAE) para conhecer os níveis de separação dos participantes em relação aos pais (Allen, Hauser, Bell, \& O'Connor, 1994; Marsh et al., 2003) ou com o objetivo de investigar a associação da autonomia emocional à consequências positivas ou negativas para o desenvolvimento (Beyers et al., 2005).

Além da autonomia comportamental e emocional, aparecem em cinco artigos conceitos gerais, compreendendo a autonomia como uma habilidade global, composta por diferentes dimensões que coexistem na relação com os outros (Liu \& Yeh, 2011; Noom et al. 2001; Reichert \& Wagner, 2007; Yeh \& Yang, 2006; Yu, 2011).

Finalmente, Lee, Beckert e Goodrich (2010) utilizam em seu artigo o conceito de autonomia cognitiva, entendendo-a como a habilidade do adolescente de pensar de forma independente. Já Brezina (2008) compreende a autonomia como "o poder sobre si mesmo, a habilidade de resistir às demandas dos outros e se envolver em ações sem a permissão dos outros" (Agnew, 1984, p. 220). Os demais artigos não apresentam clareza conceitual.

\section{Instrumentos}

Diante de tal multiplicidade de conceitos, muitos são os instrumentos utilizados para avaliar e mensurar a autonomia. 
Oito artigos utilizam entrevista e/ou observação como método de coleta de dados. Seis destes incluem tarefas de interação entre pais e filhos, sendo que cinco destes utilizam o Autonomy and Relatedness Coding System (Allen, Hauser, O'Connor, \& Bell, 2002) para codificação e análise dos dados.

Ainda são utilizadas também escalas de mensuração de diferentes dimensões e da necessidade de autonomia dos jovens (Need for Autonomy, baseada em Agnew, 1984; Feldmand \& Quatman, 1988). Além disso, sete artigos utilizam escalas para medir variáveis que podem ser observadas como demonstração de autonomia comportamental. Entre elas, as medidas de legitimidade da autoridade parental (Cumsille, Darling, \& Martínez, 2010) ou de como a tomada de decisão acontece entre pais e filhos (Decision-making questionnaire - Dornbusch et al., 1985; Family decision-making, Smetana et al., 2004).

Quatro artigos utilizam escalas de medida do apoio parental à autonomia (Family Environment Scale, Moos, 1974; CollegeStudent Scale, Robbins, 1994) e dois artigos mensuram a concessão de autonomia por parte dos pais (Parental Behavior Questionnaire, Dekovic, Wissink, \& Meijer, 2004; Perception of parents scale, Grolnick, Ryan, \& Deci, 1991). Os demais artigos não apresentaram medida para autonomia, sendo um de revisão teórica e os outros 12 que discutem resultados já obtidos, compreendendo a autonomia como independência ou individuação. O resumo dos instrumentos utilizados nos artigos e a que eles objetivavam medir encontra-se na Tabela 1.

Tabela 1

Tipo de Instrumentos Utilizados Para Medir a Autonomia, Seus Objetivos e Exemplo de Utilização
Tipo de instrumento
Instrumento
O que objetivam medir
Exemplo

Entrevistas $\quad$ Estruturadas $\quad$ Gerenciamento de informação Bakken \& Brown, 2010

Semiestruturadas

Autonomy and Relatedness Coding System

(Allen et al., 1994)

The family interaction task (Weinfield et al., 2002)
Promoção de autonomia

Willemen et al., 2010
Allen et al., 2002

Marsh et al., 2003

\section{Escalas}

Emotional Autonomy Scale (Steinberg \& Silverberg, 1986)

Autonomy Questionnaire (Noom et al., 2001ha)

Dimensões de autonomia
Cognitive Autonomy and Self

Evaluation Inventory - CASE (Beckert, Autonomia cognitiva 2007)

Adolescent Autonomy Scale (Yeh \& Yang, 2006)
Autonomia Emocional

Autonomia funcional, atitudinal e emocional
Pace \& Zappulla, 2010

Beyers et al., 2005

Lee et al., 2010

$\begin{array}{ll}\text { Autonomia individual e relacional } & \text { Liu \& Yeh, } 2011 \\ \text { Yeh \& Yang, } 2006\end{array}$
Tomada de decisão

Decision-making questionnaire (Dornbusch, 1985)
Processo de tomada de decisão

Smetana et al., 2004

Romich et al., 2009
College-Student Scale (Robbins, 1994)

Parental Behavior Questionnaire (Wissink et al., 2004)

Perceptions of Parents Scale (Grolnick et al., 1991)
Suporte parental para autonomia

Ferguson et al., 2010

Concessão de autonomia pelos pais

Scholte et al., 2001

Concessão de autonomia pelos pais
Eichelsheim et al., 2010 


\section{Variáveis}

$\mathrm{Na}$ análise dos artigos, observou-se inúmeras tentativas de responder duas questões cruciais: como se desenvolve a autonomia; e, quais as consequências do desenvolvimento dessa habilidade. Diante das dificuldades metodológicas de abarcar tal complexidade e do intrincado processo do seu desenvolvimento e construção, as pesquisas se dedicam a conhecer recortes desse constructo. Com delineamentos transversais, em sua maioria, é possível apenas apontar determinadas associações da autonomia com consequências positivas e negativas para o desenvolvimento, além de definir alguns preditores.

A fim de conhecer as principais variáveis associadas nas publicações recentes sobre a autonomia, analisou-se 37 artigos classificados como principais. A primeira constatação foi a grande quantidade de variáveis estudadas. Frente a isso, realizouse uma Análise de Conteúdo (Bardin, 2004) de tais variáveis, agrupando-as por frequência e conteúdo.

As variáveis de caracterização dos participantes são encontradas em todos os artigos pesquisados. As principais são sexo, idade, nível socioeconômico (determinado pelo salário médio da família ou pelo nível educacional e ocupação dos pais), estabilidade da família (família intacta ou não) e finalmente raça. Diversas dessas variáveis são observadas ou controladas nas análises, demonstrando a compreensão dos autores de que a autonomia, assim como o desenvolvimento humano, sofre grande influência do contexto no qual o sujeito está inserido (Bronfenbrenner, 1986).

Em algumas pesquisas, diferenças de sexo e de idade são encontradas. Meninos parecem ter mais autonomia comportamental e cognitiva do que as meninas (Daddis, 2011; Lee, Beckert, \& Goodrich, 2010) e as meninas esperam ter maior autonomia e liberdade mais tarde que os meninos (Fuligni, 1998). Sujeitos mais velhos também apresentam maiores índices de autonomia quando comparados com os mais novos (Daddis, 2011; Scabini, 2000). Outras pesquisas, por sua vez, apontam maiores índices de autonomia comportamental associado às meninas (Wray-Lake et al., 2010). Por outro lado, diversas pesquisas não encontraram diferenças entre os sexos nos níveis de autonomia em jovens afro-americanos (Smetana \& Gettman, 2006), europeu-americanos (Butner et al., 2009) italianos (Scabini, 2000) ou alemães (Seiffge-Krenke \& Pakalniskiene, 2011).

Com relação ao nível socioeconômico, para um grupo de adolescentes Coreanos essa variável aparece positivamente associada com o nível de autonomia e connectedness no primeiro tempo de um estudo longitudinal, porém, ao final de cinco anos, não é considerado preditor de mudanças nos níveis de autonomia (Yu, 2011). Em outra pesquisa que avalia a relação de risco social (caracterizada por baixo salário dos pais e localização de moradia da família) e autonomia em adolescentes americanos brancos de baixo nível socioeconômico (McElhaney \& Allen, 2001), foram encontradas associações negativas entre autonomia e nível socioeconômico. Em contextos de maior risco, os adolescentes interpretavam menores índices de autonomia como uma forma de proteção dos pais. Já em contextos de menor risco, baixos níveis de autonomia (ou seja, menor liberdade de comportamento dada pelos pais) eram considerados pelos jovens como controle psicológico.

Em outras pesquisas, porém, nenhuma das variáveis sociodemográficas é diretamente determinante para os níveis de autonomia (Allen et al., 1994; Marsh et al., 2003), apesar de serem significativas para outras variáveis como comportamento de risco, sucesso acadêmico (Smetana et al., 2004) ou autoestima (Allen et al., 1994). Dessa forma, e diante de resultados tão contraditórios, as variáveis de contexto parecem essenciais numa análise minuciosa desse fenômeno.

Além dos dados sociodemográficos, foi realizada uma categorização temática das variáveis relacionadas à autonomia apresentadas nos artigos. As variáveis foram agrupadas em subcategorias, que por sua vez, foram reagrupadas em categorias mais abrangentes. $\mathrm{O}$ ajustamento psicossocial do adolescente $\mathrm{e}$ o clima familiar foram as categorias que surgiram na análise, conforme se pode observar na tabela 2.

A categoria Ajustamento refere-se às variáveis que buscavam investigar os níveis de ajustamento psicossocial dos participantes. As principais subcategorias dizem respeito

Tabela 2

Categoria Ajustamento - Significados e Exemplos de Utilização

\begin{tabular}{|c|c|c|}
\hline Subcategoria & Significado & Exemplos \\
\hline Bem-estar & $\begin{array}{l}\text { Satisfação de vida, na escola } \\
\text { Bem-estar físico } \\
\text { Afetos positivos ou negativos }\end{array}$ & $\begin{array}{l}\text { Ferguson et al., } 2010 \\
\text { Butner et al., } 2009 \\
\text { Lekes et al., } 2010\end{array}$ \\
\hline Autoestima & $\begin{array}{l}\text { Autoestima ou } \\
\text { autoconceito positivo }\end{array}$ & $\begin{array}{l}\text { Soenens et al., } 2007 \\
\text { Allen et al., } 1994\end{array}$ \\
\hline $\begin{array}{l}\text { Problemas de } \\
\text { internalização }\end{array}$ & Depressão, Ansiedade, Ideação suicida & $\begin{array}{l}\text { Marsh et al., } 2003 \\
\text { Willemen et al., } 2010\end{array}$ \\
\hline $\begin{array}{l}\text { Problemas de } \\
\text { externalização }\end{array}$ & $\begin{array}{l}\text { Comportamento de risco*, problemas de comportamento*, } \\
\text { delinquência* e agressão }\end{array}$ & $\begin{array}{l}\text { Scholte et al., } 2001 \\
\text { Smetana et al., } 2004 \\
\text { Brezina, 2008 }\end{array}$ \\
\hline
\end{tabular}


ao bem estar, autoestima e aos problemas de internalização e externalização. Ainda são utilizadas outras variáveis como desempenho acadêmico (Fuhrman \& Holmbeck, 1995), habilidade social ou cognitiva (Allen, Marsh, et al., 2002), impulsividade (Romich et al., 2009) e hostilidade (Allen, Hauser, et al., 2002) como forma de avaliar o ajustamento dos adolescentes.

Muita autonomia, muito cedo, especialmente nos domínios multifacetado, convencional e prudencial, se associa a menor sucesso acadêmico, maiores índices de problemas de comportamento, menores índices de autovalor e maiores índices de sintomas depressivos (Smetana et al., 2004). Porém, numa análise longitudinal (cinco anos), os níveis iniciais de autonomia não foram preditores dos níveis de problemas de comportamento ao final. Por outro lado, mais autonomia no domínio pessoal se associa a maiores níveis de autovalor. De forma geral os resultados apontam que compartilhar a autonomia de decisão com os pais em domínios convencional e prudencial, se associa aos melhores índices de ajustamento (Smetana \& Gettman, 2006; Smetana et al., 2004). Altos índices de autonomia também se associam a menores índices de agressão em adolescentes Holandeses de diferentes influências culturais (Eichelsheim et al., 2010).

A categoria Clima familiar, por sua vez, incluiu variáveis que buscavam conhecer o contexto familiar do participante, assim como a influência desse contexto no desenvolvimento da autonomia, como pode-se observar na Tabela 3.

As principais subcategorias foram: relatedness e connectedness, apego, suporte parental, legitimidade da autoridade parental, conflito familiar e utilização de controle psicológico. Ainda foram incluídos afeto (Fuhrman \& Holmbeck, 1995), coesão familiar (Peterson et al., 1999), autorrevelação - disclosure (Eichelsheim et al., 2010), conformidade com as expectativas parentais (Yeh \& Yang, 2006), entre outros.

Resultados de diferentes pesquisas vêm demonstrando os efeitos protetivos de um clima familiar positivo, com maior connectedness, afeto, suporte, além de uma comunicação aberta e regras claras (Casullo \& Liporace, 2008). Pesquisa com 1023 adolescentes holandeses demonstraram que uma maior proteção parental (monitoramento) se associa a menores índices de problemas de comportamento (Sentse, Dijkstra, Lindenberg, Ormel, \& Veenstra, 2010). No que diz respeito à autonomia, diversos resultados demonstram que maiores índices de suporte parental (Liu \& Yeh, 2011; Soenens et al., 2007) e relatedness (Allen, et al., 1994) se associam com maiores índices de autonomia. A autonomia também se associa positivamente com uma maior abertura para supervisão por parte dos adolescentes (Wray-Lake et al., 2010).

O suporte parental, particularmente, tem sido apontado como positivo para o desenvolvimento da autonomia e satisfação de vida (Ferguson et al., 2010) tanto por participantes de países individualistas (Dinamarca e Estados Unidos) como por adolescentes de países coletivistas (Coréia do Sul). Além disso, essa variável se associa a menores índices de problemas de comportamento em participantes holandeses, mesmo diante de eventos estressores. Por outro lado, um clima familiar negativo, marcado por coercitividade, punição e retirada de afeto (love withdrawal) tende a ser associado a menores índices de autonomia (Peterson et al., 1999).

É importante, contudo, atentar para o instrumento utilizado para medir a autonomia e ao contexto dos participantes. Pesquisas utilizando a Escala de Autonomia Emocional (Steinberg \& Silverberg, 1986) apontam resultados diferentes dos já discutidos. Segundo Fuhrman e Holmbeck (1995), em um clima emocional positivo (afeto, coesão, monitoramento parental e baixa intensidade de conflito), altos índices de autonomia emocional se associam a um ajustamento negativo (problemas de comportamento internalizante e externalizante). Já em clima emocional negativo, o afastamento dos pais (altos índices de autonomia emocional) se associa a um ajustamento positivo.

Pode-se observar uma discussão cada vez mais presente nas pesquisas analisadas: é a autonomia uma capacidade a ser

Tabela 3

Categoria Clima Familiar - Significados e Exemplos de Utilização

\begin{tabular}{lll}
\hline \multicolumn{1}{c}{ Subcategoria } & \multicolumn{1}{c}{ Significado } & \multicolumn{1}{c}{ Exemplos } \\
\hline Relatedness ou Connectedness & $\begin{array}{l}\text { Comunicação, cuidado, confiança, aceitação, } \\
\text { expor razóes das decisões }\end{array}$ & $\begin{array}{l}\text { Smetana \& Gettman, 2006 } \\
\text { Yu, 2011 }\end{array}$ \\
Apego & Attachment & McElhaney \& Allen, 2001 \\
Suporte & Percepção de suporte parental para autonomia & Scholte et al., 2001 \\
Legitimidade da autoridade parental & Aceitação da autoridade parental & Daddis, 2011 \\
Conflito & Conflito entre pais e filhos & Fuligni, 1998 1999 \\
Controle psicológico & & Soenens et al., 2007 \\
\hline
\end{tabular}


desenvolvida através da separação e individuação (portanto, de afastamento dos pais e de desenvolvimento individual) ou de desenvolvimento concomitante com a relação próxima e afetuosa com as figuras de apego? Ferguson, Kasser e Jahng (2010) ao analisarem adolescentes americanos (descendentes de europeus, mexicanos, chineses e filipinos) afirmaram que a autonomia é uma capacidade importante para o desenvolvimento e para o bem estar, tanto em adolescentes de influência individualista ou coletivista e mesmo em relações de maior proximidade, autoridade e legitimidade parental.

\section{Discussão e conclusões}

Nessa revisão, puderam-se mapear os principais conceitos atribuídos à autonomia adolescente, seu processo de desenvolvimento e as principais variáveis associadas ao fenômeno. A construção de um panorama das publicações sobre a autonomia adolescente nos últimos 15 anos no Brasil e 20 anos no exterior, permitiu compreender melhor a importância dessa temática para a psicologia do desenvolvimento, assim como os principais focos destes estudos.

Apesar da importância da temática da autonomia, faz-se necessário apontar que o conhecimento produzido nessa área tem se baseado, em grande parte, em publicações que se referem a um adolescente específico: de 14 a 17 anos, norte americano, branco, de nível socioeconômico médio. A única publicação com amostra brasileira incluída nessa revisão trata de um perfil semelhante: jovens de 14 e 15 anos, de escolas privadas da região Sul do país e de nível socioeconômico médio e alto. Ao afirmar isso, busca-se não desvalorizar todo o conhecimento já produzido sobre essa população, mas atentar para a impossibilidade de generalização das relações e padrões descritos para este grupo. Não há como afirmar que tais padrões e variáveis sejam inteiramente adequados para outros grupos adolescentes. Dessa forma, percebe-se a necessidade de novas pesquisas sobre essa população no Brasil e com adolescentes de outros contextos (minorias étnicas, diferentes níveis socioeconômicos e educativos).

Para além dessa constatação, revisar essa literatura permitiu perceber a complexa construção conceitual ao descrever a autonomia. De forma geral, diferentes conceitos têm sido utilizados para descrever uma mesma habilidade. Por um lado, pode-se pensar que uma maior padronização dos conceitos permitiria uma comparação mais clara dos resultados encontrados nos estudos sobre a autonomia. Por outro, uma habilidade tão complexa e multifacetada como esta (tendo ainda a adolescência como palco) carece de múltiplas visões, interpretações e análises. Dessa forma, uma maior clareza conceitual ao estudar qualquer visão da autonomia (suas dimensões, suas associações e sua construção) faz-se relevante. Especificações mais explícitas sobre "que autonomia" os autores se referem, ou a que dimensão da autonomia se referem podem contribuir, não para a padronização de um único modelo de análise, mas para possibilitar uma compreensão mais sistemática e rigorosa das variáveis preditoras e consequentes do desenvolvimento dessa habilidade.

A autonomia comportamental é o conceito mais frequentemente utilizado nas publicações selecionadas, seguida pela autonomia emocional. É interessante perceber que apesar de em menor número, os artigos que trabalham com a autonomia emocional são mais homogêneos em relação à sua conceituação e à utilização da EAE (Steinberg \& Silverberg, 1986) como instrumento de mensuração. As pesquisas em autonomia comportamental, por sua vez, apresentam uma maior diversidade de instrumentos e nuances conceituais. Ainda assim, na análise dos artigos focados na Autonomia Emocional percebe-se que, apesar da padronização instrumental, alguns resultados são extremamente contraditórios. Encontra-se em diferentes trabalhos, altos índices de Autonomia Emocional como positivos (Steinberg \& Silverberg, 1986) ou como negativos para o desenvolvimento adolescente (Ryan \& Lynch, 1989). Já as pesquisas em autonomia comportamental, apesar de sua heterogeneidade conceitual e instrumental, têm encontrado resultados semelhantes no que diz respeito aos níveis de autonomia, associando altos índices dessa dimensão ao um bom ajustamento (Ferguson et al., 2010; Soenens et al., 2007), desde que sua concessão não seja precoce - early adolescence (Smetana et al., 2004).

Constatou-se o interesse dos pesquisadores da temática em conhecer as variáveis preditoras do desenvolvimento da autonomia e as possíveis consequências da autonomia no desenvolvimento e ajustamento dos jovens. As variáveis de caracterização e contexto dos participantes estão sempre presentes nos estudos, comprovando a importância e influência do contexto no qual o sujeito está inserido na compreensão do fenômeno. Ressalta-se, entretanto, que tais variáveis não identificam resultados homogêneos, o que aponta para a importância da contínua analise de tais variáveis no estudo da autonomia.

Com relação às variáveis de ajustamento associadas à autonomia, encontraram-se pesquisas sobre diferentes temáticas. Segundo os resultados encontrados, maiores índices de autonomia podem ser associados a maior autovalor e autoestima. A autonomia aparece negativamente associada com índices de agressão. O clima familiar também aparece como relevante nas pesquisas analisadas. Os resultados encontrados apontam que um clima familiar positivo, marcado por maior suporte para autonomia e maior relatedness se associam a maiores índices de autonomia. Já um clima familiar negativo, marcado por coercitividade, punição e retirada de afeto (love withdrawal) tende a ser associado a menores índices de autonomia.

Uma vertente de pesquisas que apoia esses resultados é aquela que aponta a importância de se estudar a autonomia como uma habilidade que melhor se desenvolve no contato próximo das figuras relevantes para o adolescente, como seus pais. Um crescente número de pesquisas com esse foco tem apresentado resultados demonstrando essa associação como positiva para o desenvolvimento (Kagitçibasi, 2005; Smetana \& Gettman, 2006) e tal perspectiva merece mais estudos que discriminem o tipo de relação favorecedora da autonomia.

Finalmente, diante da multiplicidade de conceitos e variáveis associados à autonomia, pode-se pensar que esta é uma habilidade complexa, marcada por inúmeras variáveis contextuais, entre elas as familiares e a do ambiente direto do sujeito. Tal multiplicidade 
abre espaço para um olhar ecológico-sistêmico (Bronfrenbrenner, 1986), que possibilite abarcar a interação desses diversos contextos, necessários para a compreensão desse fenômeno. Ao possibilitar a análise dos diferentes sistemas envolvidos no desenvolvimento da autonomia, essa visão nos permite contemplar tal complexidade de forma abrangente, mesmo diante de dificuldades metodológicas de acessa-la como um todo.

Espera-se que a partir dessa revisão sistemática, outras pesquisas possam ser realizadas sobre a temática da autonomia adolescente, especialmente no Brasil. É curioso pensar que uma temática com um número relevante de pesquisas e publicações no exterior seja tão pouco estudada no Brasil. Essa escassez talvez se deva à dificuldade de circunscrever esse construto e encontrar instrumentos adequados para medi-lo, como já foi demonstrado nas discussões anteriores. É urgente, porém, dentre os diversos temas de relevância para a adolescência, discutir a juventude em suas ações afirmativas. Apesar da relevância social de estudar os riscos da adolescência (uso e abuso de álcool e drogas, gravidez precoce, violência, entre outros) é cada vez mais importante afirmar vivências positivas e adaptativas para essa faixa etária. A autonomia, enquanto habilidade que expressa tanto a relação com o outro quanto a relação consigo mesmo, pode ser uma dessas expressões afirmativas da juventude contemporânea.

\section{Referências}

Agnew, R. (1984). Autonomy and delinquency. Sociological Perspectives, 27(2), 219-240

Allen, J. P., Hauser, S. T., Bell, K. L., \& O'Connor, T. G. (1994). Longitudinal assessment of autonomy and relatedness in adolescent-family interactions as predictors of adolescent ego development and self-esteem. Child Development, 65(1), 179-194.

Allen, J. P., Hauser, S. T., O’Connor, T. G., \& Bell, K. L. (2002). Prediction of peer-rated adult hostility from autonomy struggles in adolescent-family interaction. Development and Psychopathology, 14(1), 123-137.

Allen, J. P., Marsh, P., McFarland, C., McElhaney, K. B., Land, D. J., Jodl, K. M., \& Peck, S. (2002). Attachment and autonomy as predictors of the development of social skills and delinquency during midadolescence. Journal of Consulting and Clinical Psychology, 70(1), 56-66. doi: http://dx.doi. org/10.1037\%2F\%2F0022-006X.70.1.56

Anderson, R. A., Worthington, L., Lowell, A., William, T., \& Jennings, G. (1994). The development of an autonomy scale. Contemporary Family Therapy, 16(4), 329-345.

Bakken, J. P., \& Bronw, B. B. (2010). Adolescent secretive behavior: African American and Hmong adolescents' strategies and justifications for managing parent's knowledge about peers. Journal of Research on Adolescence, 20(2), 359-388. doi: 10.1111/j.1532-7795.2010.00642.x

Bardin, L. (2004). Análise de conteúdo. Lisboa: Edições 70.

Baumrind, D. (1966). Effects of authoritative parental control on child behavior. Child Development, 37(4), 887-907.

Beckert, T. (2007). Cognitive autonomy and self evaluation in adolescence: A conceptual investigation and instrument development. North American Journal of Psychology, 9(December), 579-594.

Beyers, W., Goossens, L., van Calster, B., \& Duriez, B. (2005). An alternative substantive factor structure of the Emotional Autonomy Scale. European Journal of Psychological Assessment, 21(3), 147-155. doi: 10.1027/10155759.21.3.147

Blair, B. L., \& Fletcher, A. C. (2011). "The only 13-year-old on planet earth without a cell phone": meanings of cell phones in early adolescents" everyday lives. Journal of Adolescent Research, 26(2), 155-177. doi:
$10.1177 / 0743558410371127$

Blos, P. (1994). Adolescência: Uma interpretação psicanalítica. São Paulo, SP: Martins Fontes

Brezina, T. (2008). Recognition denial, need for autonomy, and youth violence. New Directions for Youth Development, 119(Fall), 111-128.

Bronfenbrenner, U. (1986). Ecology of the family as a context for human development: research perspectives. Developmental Psychology, 22(6), 723-742. doi: 10.1037/0012-1649.22.6.723

Butner, J., Berg, C. A., Osborn, P., Butter, J. M., Godri, C., Fortenberry, K. T., ... Wiebe, D. J. (2009). Parent-adolescent discrepancies in adolescent's competence and the balance of adolescent autonomy and adolescent and parent well-being in the context of type 1 Diabetes. Developmental Psychology, 45(3), 835-849. doi: 10.1037/a0015363

Casullo, M. M., \& Liporace, M. F. (2008). Percepción sobre estilos e inconsistencias parentales en adolescentes argentinos. Estudos de Psicologia (Campinas), 25(1), 3-9. doi: doi.org/10.1590/S0103-166X2008000100001

Celen, N., Cok, F., Bosma, H. A., \& Djurre, H. Z. (2006). A percepção de adolescentes e de pais sobre decisão e autonomia. Paidéia, 16(35), 349-363.

Cumsille, P., Darling, N., \& Martínez, M. L. (2010). Shading the truth: the patterning of adolescents' decisions to avoid issues, disclose, or lie to parents. Journal of Adolescence, 33(2), 285-296. doi: 10.1016/j. adolescence. 2009.10 .008

Daddis, C. (2011). Desire for increased autonomy and adolescents' perceptions of peer autonomy: "Everyone else can; why can't I?" Child Development, 82(4), 1310-1326. doi: 10.1111/j.1467-8624.2011.01587.x

Deci, E. L., \& Ryan, R. M. (2000). The "what" and "why" of goal pursuits: Human needs and the self-determination of behavior. Psychological Inquiry, 11, 319-338.

Dekovic M., Wissink I. B., \& Meijer, A. M. (2004). The role of family and peer relations in adolescent antisocial behavior: Comparison of four ethnic groups. Journal of Adolescence, 27, 497-514

Dornbusch, S. M., Carlsmith, J. M., Bushwall, S. J., Ritter, P. L., Leiderman, H., Hastorf, A. H., \& Gross, R. T. (1985). Single parents, extended households, and the control of adolescents. Child Development, 56(2), 326-341. doi: $10.2307 / 1129723$

Eichelsheim, V. I., Buist, K. L, Dekovic, M., Wissink, I. B., Frijns, T., van Lier, P. A., ... Meeus, W. H. (2010). Associations among the parent-adolescent relationship, aggression and delinquency in different ethnic groups: A replication across two Dutch samples. Social Psychiatry and Psychiatric Epidemiology, 45(3), 293-300. doi: 10.1007/s00127-009-0071-z

Feldman, S. S., \& Quartman, T. (1988). Factors influencing age expectations for adolescent autonomy: A study of early adolescents and parents. Journal of Early Adolescence, 8(4), 325-342. doi: 10.1177/0272431688084002

Ferguson, Y. L., Kasser, T., \& Jahng, S. (2010) Differences in life satisfaction and school satisfaction among adolescents from three nations: the role of perceived autonomy support. Journal of Research on Adolescence, 21(3), 649-661. doi: 10.1111/j.1532-7795.2010.00698.x

Fuhrman, T., \& Holmbeck, G. N. (1995). A contextual-moderator analysis of emotional autonomy and adjustment in adolescence. Child Development, 66(3), 793-811.

Fuligni, A. J. (1998). Authority, autonomy, and parent-adolescent conflict and cohesion: A study of adolescents from Mexican, Chinese, Filipino, and European backgrounds. Developmental Psychology, 34(4), 782-792. doi:10.1037/0012-1649.34.4.782

Grolnick, W. S., Ryan, R. M., \& Deci, E. (1991). Inner resources for school achievement: Motivational mediators of children's perceptions of their parents. Journal of Educational Psychology, 83(4), 508-517. doi: 10.1037/0022-0663.83.4.508

Haase, C. M., Tomasik, M. J., \& Silbereisen, R. K. (2008) Premature behavioral autonomy: Correlates in late adolescence and young adulthood. European Psychologist, 13(4), 255-266. doi: 10.1027/1016-9040.13.4.255

Jamabo, A., \& Jamabo, T. (2010). Filial relationship and autonomy of senior 
secondary school students in Rivers State. Bangladesh e-Journal of Sociology, 7(2), 42-49.

Kagitçibasi, C. (2005) Autonomy and relatedness in cultural context: Implications for self and family. Journal of Cross-Cultural Psychology, 36(4), 403-422.

Lee, G., Beckert, T. E., \& Goodrich, T. R. (2010). The relationship between individualistic, collectivistic, and transitional cultural value orientation and adolescent's autonomy and identity status. Journal of Youth and Adolescence, 39(8), 882-893. doi: 10.1007/s10964-009-9430-Z

Lekes, N., Gingras, I., Philippe, F. L., Koestner, R., \& Fang, J. (2010). Parental autonomy-support, intrinsic life goals, and well-being among adolescents in China and North America. Journal of Youth and Adolescence, 39(8), 858-869. doi: 10.1007/s10964-009-9451-7

Liu, Y., \& Yeh, K. (2011). The mediating effect of mother-adolescent interaction on the relationship between maternal ego development and adolescent individuation in Taiwan. Swiss Journal of Psychology, 70(3), 155-164. doi: 10.1024/1421-0185/a000051

Marsh, P., McFarland, F. C., Allen, J. P., McElhaney, K. B., \& Land, D. (2003). Attachment, autonomy, and multifinality in adolescent internalizing and risky behavioral symptoms. Development and Psychopathology, 15(2), 451-467. doi: 10.1017\%2FS0954579403000245

McElhaney, K. B., \& Allen, J. P. (2001). Autonomy and adolescent social functioning: the moderating effect of risk. Child Development, 72(1), 220235. doi: 10.1111/1467-8624.00275

Moos, R. H. (1974). Family Environment Scale (FES) Preliminary manual. Palo Alto, CA: Social Ecology Laboratory, Department of Psychiatry, Stanford University.

Noon, M. J., Dekovic, M., \& Meeus, W. (2001) Conceptual Analysis and Measurement of Adolescent Autonomy. Journal of Youth and Adolescence, 30(5), 577-595. doi: 10.1023/A:1010400721676

Pace, U., \& Zappulla, C. (2010). Relations between suicidal ideation, depression, and emotional autonomy from parents in adolescence. Journal of Child and Family Studies, 19(6), 747-756. doi: 10.1007/s10826-010-9364-9

Peterson, G. W., Bush, K. R., \& Supple, A. (1999). Predicting adolescent autonomy from parents: relationship connectedness and restrictiveness. Sociological Inquiry, 69(3), 431-457. doi: 10.1111/j.1475-682X.1999.tb00880.x

Reichert, C. B., \& Wagner, A. (2007). Autonomia na adolescência e sua relação com os estilos parentais. Psico, 38(3), 292-299. Recuperado de http://tede. pucrs.br/tde_busca/arquivo.php?codArquivo $=223$

Robbins, R. J. (1994). An assessment of perceptions of parental autonomy support and control: child and parent correlates (Tese de doutorado não publicada). Departamento de Psicologia, University of Rochester, Rochester, Nova Iorque.

Romich, J., Lundberg, S., \& Tsang, K. P. (2009). Independence giving or autonomy taking? Childhood predictors of decision-making sharing patterns between young adolescents and parents. Journal of Research on Adolescence, 19(4), 587-600. doi: 10.1111\%2Fj.1532-7795.2009.00612.x

Ryan, R. M., \& Lynch, J. H. (1989). Emotional autonomy versus detachment: Revisiting the vicissitudes of adolescence and young adulthood. Child Development, 60(2), 340-356. . doi:10.2307/1130981
Scabini, E. (2000). Parent-child relationships in Italian families: connectedness and autonomy in the transition to adulthood. Psicologia: Teoria e Prática, 16(1), 23-30. doi: 10.1590/S0102-37722000000100004

Scholte, R. H. J., van Lieshout, C. F. M., \& van Aken, M. A. G. (2001). Perceived relational support in adolescence: Dimensions, configurations, and adolescent adjustment. Journal of Research on Adolescence, 11(1), 71-94. doi: 10.1111/1532-7795.00004

Seiffge-Krenke, I., \& Pakalniskiene, V. (2011). Who shapes whom in the family: Reciprocal links between autonomy support in the family and parent's and adolescents' coping behaviors. Journal of Youth and Adolescence, 40(8), 983-995. doi: 10.1007/s10964-010-9603-9

Sellers, K., Black, M. M., Boris, N. W., Oberlander, S. E., \& Myers, L. (2011) Adolescent mother's relationships with their own mothers: Impact on parenting outcomes. Journal of Family Psychology, 25(1), 117-126. doi: $10.1037 / \mathrm{a} 0021877$

Sentse, M., Dijkstra, J. K., Lindenberg, S., Ormel, J., \& Veenstra, R. (2010). The delicate balance between parental protection, unsupervised wandering, and adolescents' autonomy and its relation with antisocial behavior: The TRAILS study. International Journal of Behavioral Development, 34(2), 159-167. doi: 10.1177/0165025409350949

Slater, M. D., Kelly, K. J., Lawrence, F. R., Stanley, L. R., \& Comello, M. L. G. (2011). Assessing media campaigns linking marijuana non-use with autonomy and aspirations: "Be under your own influence" and ONDCP's "Above the influence". Prevention Science, 12(1), 12-22.

Smetana, J. G., \& Gettman, D. C. (2006). Autonomy and relatedness with parents and romantic development in African American adolescents. Developmental Psychology, 42(6), 1347-1351. doi: 10.1037/0012-1649.42.6.1347

Smetana, J. G., Campione-Barr, N., \& Daddis, C. (2004). Longitudinal development of family decision making: Defining healthy behavior autonomy for middle-class African American adolescents. Child Development, 75(5), 1418-1434. doi: 10.1111/j.1467-8624.2004.00749.x

Soenens, B., Vansteenkiste, M., Lens, W., Luyckx, K., Goossens, L., Beyers,W., \& Ryan, R. M. (2007). Conceptualizing parental autonomy support: Adolescent perceptions of promotion of independence versus promotion of volitional functioning. Developmental Psychology, 43(3), 633-646. doi: 10.1037/0012-1649.43.3.633

Steinberg, L., \& Silverberg, S. B. (1986). The vicissitudes of autonomy in early adolescence. Child Development, 57(1), 841-851. doi:10.2307/1130361

Wray-Lake, L., Crouter, A. C., \& McHale, S. M. (2010). Developmental patterns in decision-making autonomy across middle childhood and adolescence: European American parents' perspective. Child Development, 81(2), 636651.

Yeh, K., \& Yang, Y. (2006). Construct validation of individuation and relating autonomy orientations in culturally Chinese adolescents. Asian Journal of Social Psychology, 9(2), 148-160. doi: 10.1111/j.1467-839X.2006.00192.x

Yu, J. J. (2011) Reciprocal associations between connectedness and autonomy among Korean adolescents: Compatible or antithetical? Journal of Marriage and Family, 73(4), 691-888. doi: 10.1111/j.1741-3737.2011.00839.x 
Paola Vargas Barbosa, Mestre em Psicologia pela Universidade Federal do Espírito Santo (UFES), é doutoranda em Psicologia no Programa de Pós-Graduação em Psicologia da Universidade Federal do Rio Grande do Sul (UFRGS). Endereço para correspondência: R. Ramiro Barcelos, 2600

Instituto de Psicologia - Sala 126, Porto Alegre - RS 90035-003. Telefone: (51) 3308-5322. E-mail: paolavargasbarbosa@gmail.com

Adriana Wagner, Doutora em Psicologia Social pela Universidad Autónoma de Madrid (UAM)/ Espanha, pós-doutora em Psicologia pela Universitat de Girona (UDG)/ Espanha, é professora adjunta do Instituto de Psicologia e do Programa de Pós-Graduação em Psicologia da UFRGS e coordenadora do Núcleo de Pesquisa Dinâmica das Relações Familiares. E-mail:

adrianawagner.ufrgs@hotmail.com 